\title{
Urinary CXCL10 Chemokine Is Associated With Alloimmune and Virus Compartment-Specific Renal Allograft Inflammation
}

\author{
Julie Ho, MD, ${ }^{1,2}$ Stefan Schaub, MSc, MD, ${ }^{3}$ Chris Wiebe, MSc, MD, ${ }^{1}$ Ang Gao, ${ }^{2}$ Caroline Wehmeier, MD, ${ }^{3}$
} Michael T. Koller, MD, ${ }^{3,4}$ Hans H. Hirsch, MD, ${ }^{5,6}$ Helmut Hopfer, MD, ${ }^{7}$ Peter Nickerson, MD, ${ }^{1,2,8}$ and Patricia Hirt-Minkowski, MD ${ }^{3}$

Background. Urinary CXC chemokine ligand 10 (CXCL10) is a promising biomarker for subclinical tubulointerstitial inflammation, but limited data exist regarding its correlation with (micro)vascular inflammation. Furthermore, no study has evaluated whether concomitant serum CXCL10 improves the discrimination for (micro)vascular inflammation. Methods. We investigated whether serum/urinary CXCL10 reflect subclinical inflammation within different renal compartments. Patients $(n=107)$ with 107 surveillance biopsies were classified as: normal histology $(n=47)$, normal histology with polyomavirus BK (BKV) or cytomegalovirus $(C M M)$ viremia $(n=17)$, moderate-severe tubulointerstitial inflammation (tubulitis $\geq 2, n=18$ ), pure microvascular inflammation $(n=15)$, and isolated $v$ lesions ( $n=10)$. Serum and urinary CXCL10 Enzyme-linked Immunosorbent Assay was performed. An independent validation set was evaluated for urine CXCL10: normal histology $(n=14)$, normal histology with BKV or CMV viremia $(n=19)$, tubulitis $\geq 2$ ( $n=15)$, pure microvascular inflammation $(n=41)$, and isolated v lesions $(n=14)$. Results. Elevated urinary CXCL10 reflected inflammation within the tubulointerstitial (urinary CXCL10/creatinine, $1.23 \mathrm{ng} / \mathrm{mmol}$ vs $0.46 \mathrm{ng} / \mathrm{mmol} ; P=0.02$; area under the curve, $0.69 ; P=0.001$ ) and $\mathrm{mi}-$ crovascular compartments (urinary CXCL10/creatinine, $1.72 \mathrm{ng} / \mathrm{mmol}$ vs $0.46 \mathrm{ng} / \mathrm{mmol} ; P=0.03$; area under the curve, 0.69 ; $P=0.02)$ compared to normal histology. Intriguingly, urinary CXCL10 was predominantly elevated with peritubular capillaritis, but not glomerulitis $(P=0.04)$. Furthermore, urinary CXCL10 corresponded with BKV, but not CMV viremia $(P=0.02)$. These urine CXCL10 findings were confirmed in the independent validation set. Finally, serum CXCL10 was elevated with BKV and CMV viremia but was not associated with microvascular or vascular inflammation $(P \geq 0.19)$. Conclusions. Urinary CXCL10 reflects subclinical inflammation within the tubulointerstitial and peritubular capillary spaces, but not the vascular/systemic compartments; this was consistent with BKV (tubulointerstitial) and CMV viremia (systemic). Serum CXCL10 was not a useful marker for (micro)vascular inflammation.

(Transplantation 2018;102: 521-529)

W ith the use of currently available immunosuppressive regimens, renal allograft rejection frequently presents as a "smoldering" process, not immediately compromising renal function, that is, subclinical rejection. This is important because persisting subclinical tubulointerstitial inflammation can induce progressive interstitial fibrosis and tubular atrophy,

Received 15 March 2017. Revision received 21 July 2017.

Accepted 15 August 2017

1 Transplantation and Nephrology, University of Manitoba, Winnipeg, Manitoba, Canada.

${ }^{2}$ Manitoba Centre for Proteomics and Systems Biology, Faculty of Medicine, University of Manitoba, Winnipeg, Manitoba, Canada.

${ }^{3}$ Transplantation Immunology and Nephrology, University Hospital Basel, Basel, Switzerland.

${ }^{4}$ Institute for Clinical Epidemiology and Biostatistics, University Hospital Basel, Basel, Switzerland.

${ }^{5}$ Infectious Diseases and Hospital Epidemiology, University Hospital Basel, Basel, Switzerland.

${ }^{6}$ Transplantation and Clinical Virology, Department of Biomedicine, University Hospital Basel, Basel, Switzerland.

${ }^{7}$ Institute for Pathology, University Hospital Basel, Basel, Switzerland.

${ }^{8}$ Diagnostic Services of Manitoba, Winnipeg, Manitoba, Canada. which ultimately reduces renal allograft survival. ${ }^{1-3}$ Furthermore, subclinical microvascular inflammation (MVI) precedes clinical antibody-mediated rejection (AMR) and is associated with a poor prognosis. ${ }^{4,5}$ Thus, detection of subclinical

This study was supported by the Else Kröner-Fresenius-Stiftung (grant 2012_A255). P.H.-M. is supported by the Astellas foundation for biomedical research (grant CH-02-RG-248); J.H. is supported by the Canadian Institutes of Health Research (grant 287559), and a CIHR New Investigator Salary Award. P.N. is supported by the Flynn Family Chair in Renal Transplantation.

The authors declare no conflicts of interest.

P.H.-M., J.H., P.N., S.S. designed the research/study. P.H.-M., J.H., A.G., S.S. performed the research/study. P.H.-M., H.H., S.S., C.W., and C.A.W. collected the data. P.H.-M., J.H., M.T.K., S.S. analyzed the data. All the authors wrote the article.

Correspondence: Patricia Hirt-Minkowski, Clinic for Transplantation Immunology and Nephrology University Hospital Basel Petersgraben 44031 Basel Switzerland. (patricia.hirt-minkowski@usb.ch).

Supplemental digital content (SDC) is available for this article. Direct URL citations appear in the printed text, and links to the digital files are provided in the HTML text of this article on the journal's Web site (www.transplantjournal.com).

Copyright ( 2017 Wolters Kluwer Health, Inc. All rights reserved.

ISSN: 0041-1337/18/10203-0521

DOI: 10.1097/TP.00000000000001931 
tubulointerstitial and (micro)vascular inflammation may provide an early therapeutic window to treat alloimmunerelated injury.

Subclinical rejection can currently only be detected by surveillance biopsies, which are costly, inconvenient, and carry a small risk of complications. ${ }^{6}$ Furthermore, sampling error may bias the histopathologic diagnosis. Unfortunately, standard monitoring (ie, serum creatinine and proteinuria) is ineffective at detecting subclinical rejection. Although serial monitoring for the development of de novo donor-specific antibody (DSA) in kidney allograft recipients may predate clinical AMR, ${ }^{4}$ its utility as an early marker for subclinical MVI is limited by cost. Taken together, these data suggest that a noninvasive, cost-efficient biomarker that correlates with subclinical tubulointerstitial and (micro)vascular inflammation would be useful to identify patients at high risk of rejection who should undergo allograft biopsy.

Urinary CXC-receptor 3 chemokines (eg, CXC chemokine ligand [CXCL]9 and CXCL10) have been evaluated as promising noninvasive biomarkers for subclinical and clinical tubulointerstitial inflammation in renal transplantation, ${ }^{7-12}$ but limited data exist regarding its correlation with (micro)vascular inflammation. Notably, in an unselected, prospective cohort, urinary CXCL10 failed to detect subclinical isolated vascular inflammation (ie, glomerulitis, peritubular capillaritis, and endarteritis). ${ }^{13}$ Conversely, other studies have shown that elevated urinary CXCL10 may be associated with MVI. ${ }^{14,15}$ Therefore, we sought to delineate the reasons for these conflicting data and speculated that CXCL10 may reflect inflammation within different renal compartments. The overall objective of this study was to characterize subclinical compartment-specific inflammation with concomitant serum and urinary CXCL10 measurements and to identify compartment-specific confounders for their use as noninvasive screening biomarkers.

\section{MATERIALS AND METHODS}

\section{Patient Population}

The study protocol was approved by the University of Basel Ethics committee, and all the participants gave informed consent. For the subclinical discovery cohort, patients were selected from a prospective, observational adult renal transplant cohort. ${ }^{13}$ Inclusion criteria were a scheduled surveillance biopsy between 3 and 12 months posttransplant meeting predefined histological criteria described below, with corresponding serum and urine samples. Specifically, all surveillance biopsies with isolated (micro)vascular inflammation without tubulitis and normal histology with concomitant infection were identified. Controls with normal histology and moderate-severe tubulointerstitial inflammation (tubulitis $\geq 2$ ) were then randomly selected in a 1:1 manner. Thus, 107 patients contributed 107 biopsies obtained at $3(\mathrm{n}=52), 6$ $(\mathrm{n}=47)$, or 12 months $(\mathrm{n}=8)$ posttransplant. Immunosuppression was selected based on the presence of pretransplant DSA, ABO blood group compatibility and HLA matching as described previously. ${ }^{16}$

The independent validation set consisted of patients from 2 prospective, observational adult renal transplant cohorts (Basel/Switzerland ${ }^{13}$ and Manitoba/Canada ${ }^{4,12}$ ). Inclusion criteria were a clinical indication or surveillance biopsy meeting the same histological criteria as the subclinical discovery cohort with a corresponding urine sample. All biopsies with isolated (micro)vascular inflammation without tubulitis and normal histology with concomitant infection were identified. Controls with normal histology and moderate-severe tubulointerstitial inflammation (tubulitis $\geq 2$ ) were then randomly selected and added. The exclusion criterion was any overlap with the subclinical discovery cohort. Thus, 103 patients contributed 103 biopsies with corresponding urine samples.

\section{Renal Allograft Histology}

Surveillance biopsies were performed with a 16 -gauge needle at 3,6 , or 12 months posttransplant as center-specific standard of care. Clinically indicated allograft biopsies were performed when serum creatinine increased by more than $20 \%$ from baseline. Histology was evaluated by light microscopy, immunofluorescence (C4d, HLA-DR), and immunohistochemistry (IHC of SV40 large T antigen), scored by the Banff schema, ${ }^{17}$ and grouped as:

\section{Subclinical Discovery Cohort}

(1) Normal histology ( $\mathrm{n}=47)$ : t0 i0 g0 v0 ptc0, C4d-

(2) Normal histology with concomitant infection $(\mathrm{n}=17)$ : $\mathrm{t} 0 \mathrm{i} 0$ $1 \mathrm{~g} 0 \mathrm{v} 0 \mathrm{ptc} 0, \mathrm{C} 4 \mathrm{~d}-/+$, IHC of SV40 large T antigen negative

(a) Polyomavirus BK (BKV) viremia $(\mathrm{n}=9)$

(b) Cytomegalovirus (CMV) viremia $(\mathrm{n}=8)$

(3) Moderate-severe tubulointerstitial inflammation with tubulitis $\geq 2(\mathrm{n}=18): \mathrm{t} \geq 2 \mathrm{i} 1-3 \mathrm{~g} 0 \mathrm{v} 0 \mathrm{ptc} 0, \mathrm{C} 4 \mathrm{~d}-$

(4) Vascular inflammation $(\mathrm{n}=25)$ :

(a) Pure MVI ( $\mathrm{n}=15)$ : t0 i0 g0-3 ptc0-3 v0, with the sum of $\mathrm{g}+\mathrm{ptc} \geq 1, \mathrm{C} 4 \mathrm{~d}+/-$

(b) Isolated v lesions $(\mathrm{n}=10)$ : t0 i0 g0 ptc0 v $\geq 1, \mathrm{C} 4 \mathrm{~d}-$

\section{Independent Validation Set}

(1) Normal histology ( $\mathrm{n}=14)$ : t0 i0 g0 v0 ptc0, C4d-

(2) Normal histology with concomitant infection $(\mathrm{n}=19)$ : t0 i0 g0 v0 ptc0, C4d-, IHC of SV40 large T antigen negative

(a) BKV viremia $(\mathrm{n}=8)$

(b) CMV viremia $(\mathrm{n}=11)$

(3) Moderate-severe tubulointerstitial inflammation with tubulitis $\geq 2(\mathrm{n}=15): \mathrm{t} \geq 2$ i1-3 g0 v0 ptc0, C4d-

(4) Vascular inflammation $(\mathrm{n}=55)$ :

(a) Pure MVI ( $\mathrm{n}=41)$ : t0 i0 g0-3 ptc0-3 v0, with the sum of $\mathrm{g}+\mathrm{ptc} \geq 1, \mathrm{C} 4 \mathrm{~d}+/-$

(b) Isolated v lesions ( $\mathrm{n}=14)$ : t0 i0 g0 ptc0 v $\geq 1$, C4d-

\section{BKV and CMV Viremia}

Screening for active BKV infection was performed by urine cytology (ie, decoy cells) and confirmed with plasma BKV real-time PCR. BKV viremia was defined as detectable BKV replication ( $\geq 100$ copies $/ \mathrm{mL})$ at the time of biopsy. Routine CMV screening was performed with real-time PCR. CMV viremia was defined as any detectable CMV replication (ie, $\geq 137 \mathrm{IU} / \mathrm{mL}$ ) at the time of the biopsy. All individuals 
in the CMV viremia group had CMV replication for at least 2 weeks.

\section{Urine and Serum Preparation}

Serum and midstream clean catch urines were collected immediately before biopsy. Serum and urines were centrifuged at $1750 \mathrm{~g}$ for 10 minutes. Serum and urine supernatants were aliquoted and stored at $-75^{\circ} \mathrm{C}$ without preservatives for future analysis.

\section{Urinary and Serum CXCL10 Measurement}

Urinary CXCL10 was retrospectively analyzed with a sandwich ELISA as previously described. ${ }^{13}$ To correct for dilutional factors, CXCL10 is expressed in relation to urine creatinine (ie, ng protein/mmol creatinine). Serum CXCL10 measurements were retrospectively performed with a sandwich ELISA. Briefly, plates were coated overnight at $4^{\circ} \mathrm{C}$ with $0.1 \mu \mathrm{g} / \mathrm{mL}$ rabbit antihuman CXCL10 polyclonal antibody (Peprotech, catalog 500-P93), washed, and then blocked. Serum samples $(50 \mu \mathrm{L})$ were performed in duplicate at neat, 1:2, $1: 4,1: 8$, and 1:16 dilutions, incubated overnight at $4^{\circ} \mathrm{C}$, and then washed. Biotinylated rabbit antihuman CXCL10 secondary polyclonal antibody (Peprotech, catalog 500-P93Bt) was added at $0.025 \mu \mathrm{g} / \mathrm{mL}$ and incubated overnight at $4^{\circ} \mathrm{C}$. Plates were then washed, developed with streptavidin-alkaline phosphatase/p-nitrophenyl phosphate (1:3000), and read at 405 nm/690 nm (Biotek Synergy 4 microplate reader, Gen 5 software, Fisher Scientific). The intra-assay and interassay coefficients of variation were $3.8 \%$ and $7.4 \%$, respectively. The detection limit of the ELISA assay was $0.975 \mathrm{pg} / \mathrm{mL}$.

\section{Statistical Analysis}

We used JMP software version 13.0.0 (SAS Institute Inc., Cary, NC) for statistical analysis. For categorical data, Fisher exact test or Pearson $\chi^{2}$ test was used and data presented as counts and percentages. Parametric continuous data were analyzed by Student $t$ tests. Nonparametric continuous data were summarized as median (interquartile range [IQR]) unless stated otherwise and analyzed by the Wilcoxon rank-sum or KruskalWallis rank sum tests. Receiver operating characteristic analyses were performed to evaluate the diagnostic performance of urinary CXCL10 for the detection of subclinical tubulointerstitial inflammation and MVI, respectively. A 2-tailed $P$ value less than 0.05 was considered statistically significant.

\section{RESULTS}

\section{Patient Characteristics}

The demographic data of the 107 patients with surveillance biopsies stratified by histological groups are summarized in Table 1 . The tubulitis $\geq 2$ group consisted of moderate $(\mathrm{t} 2 ; \mathrm{n}=14)$ to severe tubulitis $(\mathrm{t} 3 ; \mathrm{n}=4)$ with concomitant mild to severe interstitial inflammation (i1-3). The pure MVI group included AMR C4d+ $(\mathrm{n}=7)$ and AMR $\mathrm{C} 4 \mathrm{~d}-(\mathrm{n}=8)$; and the isolated $\mathrm{v}$ lesions group included Banff IIA C4d- $(n=10)$ acute rejection, respectively. Not surprisingly, there was a higher proportion of patients with pretransplant DSA $(P<0.0001)$ and receiving induction therapy with polyclonal antithymocyte globulin $+/-$ intravenous immunoglobulins (ATG + /- IVIg) $(P=0.007)$ in the pure MVI group. The groups demonstrated significant differences in Banff scores by definition (all $P$ values $<0.0001$ ).
In addition, the estimated glomerular filtration rate (eGFR) was different across the 5 groups $(P$ overall $=0.01)$. This was mainly due to lower eGFR in the infection versus normal histology group $(P=0.001)$. There were no eGFR differences in normal histology versus tubulitis $\geq 2(56 \mathrm{~mL} / \mathrm{min}$ [46-67] vs $56 \mathrm{~mL} / \mathrm{min}$ [39-72], $P=0.96)$, and normal histology versus (micro) vascular inflammation $(P=0.07)$, emphasizing the subclinical nature of the findings. Furthermore, proteinuria was not statistically different among the groups $(P=0.16)$. C-reactive protein (CRP) was statistically different between the groups; however, all values were within the normal range for CRP (reference range, $<10.0 \mathrm{mg} / \mathrm{mL}$ ). Median BKV-viremia was 4550 copies/mL (IQR, 441-185 352), median CMV-viremia was 7371 copies/mL (IQR, 6574-8648).

The independent validation set ( $\mathrm{n}=103$ patients) had very similar characteristics as the subclinical discovery cohort, and their demographic data are summarized in Table S1, SDC (http://links.lww.com/TP/B479). Interestingly, no difference in eGFR was observed between groups, despite the predominance of clinical indication biopsies in the independent validation set.

\section{Urinary CXCL10 Correlates With Subclinical Tubulointerstitial Inflammation and Pure MVI}

To investigate compartment-specific inflammation, we evaluated the correlation of concomitant urinary and serum CXCL10 levels with subclinical histopathology. Elevated urinary CXCL10 reflected inflammation within the tubulointerstitial (tubulitis $\geq 2$ vs normal histology: median urinary CXCL10/creatinine [IQR], $1.23 \mathrm{ng} / \mathrm{mmol}$ [0.48$5.23 \mathrm{ng} / \mathrm{mmol}]$ vs $0.46 \mathrm{ng} / \mathrm{mmol}[0.32-1.31 \mathrm{ng} / \mathrm{mmol}] ; P=$ 0.02 ), and pure microvascular compartment (pure MVI vs normal histology: median urinary CXCL10/creatinine [IQR], $1.72 \mathrm{ng} / \mathrm{mmol}[0.50-2.73 \mathrm{ng} / \mathrm{mmol}]$ vs $0.46 \mathrm{ng} / \mathrm{mmol}[0.32-$ $1.31 \mathrm{ng} / \mathrm{mmol}] ; P=0.03$ ), respectively (Figure $1 \mathrm{~A})$. Notably, urinary CXCL10 was not associated with subclinical isolated vascular inflammation (isolated $\mathrm{v}$ lesions vs normal histology: median urinary CXCL10/creatinine [IQR], $0.64 \mathrm{ng} / \mathrm{mmol}$ $[0.36-3.69 \mathrm{ng} / \mathrm{mmol}]$ vs $0.46 \mathrm{ng} / \mathrm{mmol}[0.32-1.31 \mathrm{ng} / \mathrm{mmol}]$; $P=0.14)$ (Figure 1A).

Conversely, elevated serum CXCL10 was not associated with inflammation within the microvascular and vascular compartments $(P \geq 0.19)$, although it correlated with tubulitis (tubulitis $\geq 2$ vs normal histology: median [IQR] serum CXCL10, $64.5 \mathrm{pg} / \mathrm{mL}$ [46.4-88.1 pg/mL] vs $41.4 \mathrm{pg} / \mathrm{mL}$ [21.9-69.0 pg/mL], respectively; $P=0.01$ ) (Figure 1B). Further, urinary and serum CXCL10 levels were not associated with interstitial fibrosis and tubular atrophy and chronic (micro)vascular lesions (data not shown).

Next, we evaluated the diagnostic performance of urinary CXCL10 for detection of subclinical tubulointerstitial inflammation and pure MVI. The AUCs to discriminate subclinical tubulointerstitial inflammation and pure MVI versus normal histology were both $0.69(P=0.001$ and $P=0.02$, respectively). Combining urinary CXCL10 with pretransplant DSA did not strikingly improve the diagnostic performance for the detection of pure MVI (AUC, 0.75).

Furthermore, in a supplementary analysis, we compared urinary and serum CXCL10 in patients with isolated peritubular capillaritis ( $\mathrm{n}=8$, ptc1-3, g0, t0, i0, v0) and isolated glomerulitis $(\mathrm{n}=4, \mathrm{~g} 1, \mathrm{ptc} 0, \mathrm{t} 0, \mathrm{i} 0, \mathrm{v} 0)$ to delineate if there were any compartment-specific differences. Patients 


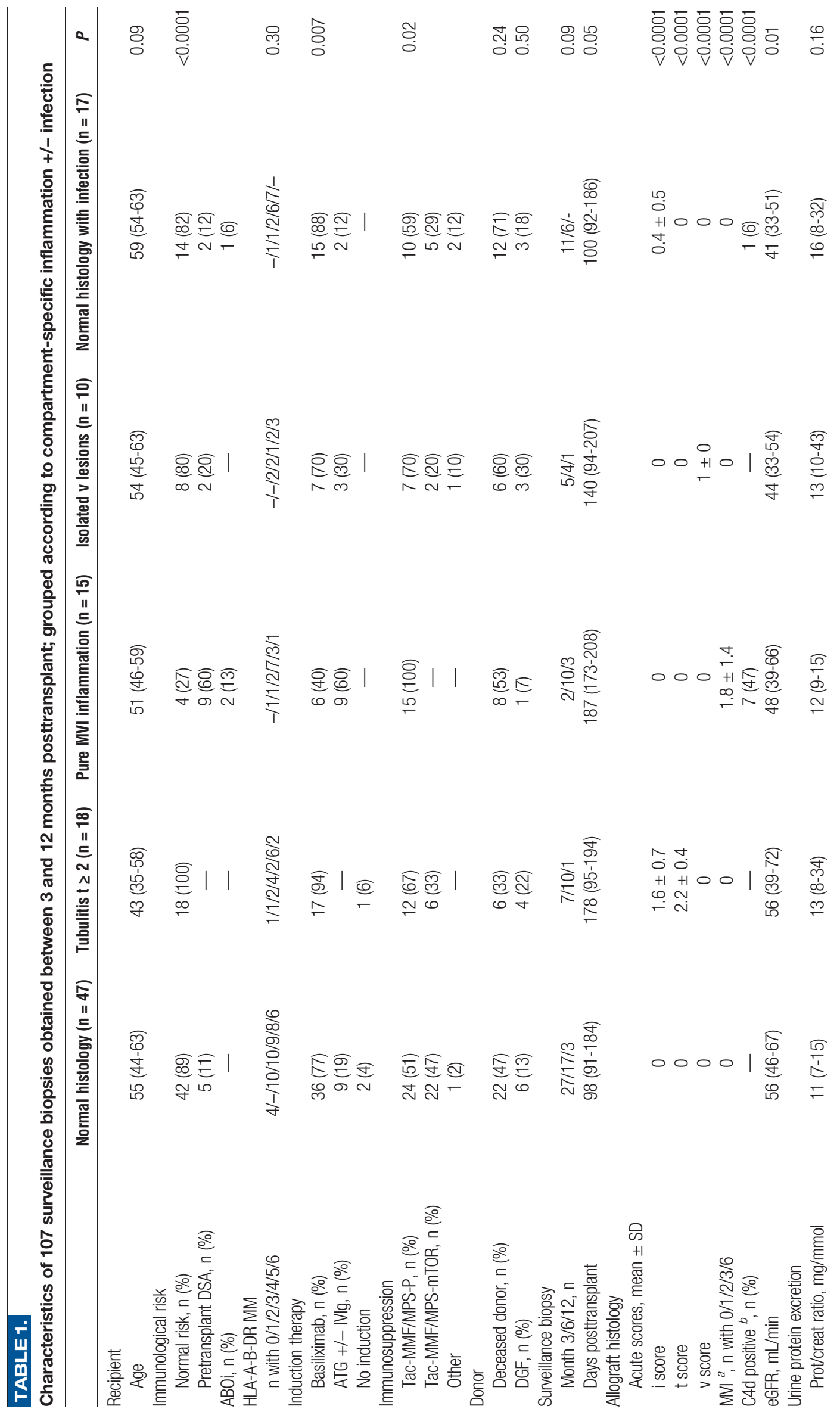




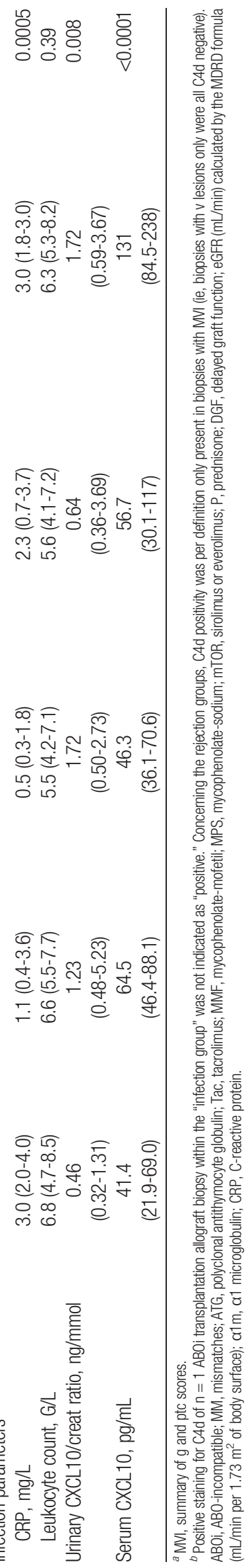

with a mixed phenotype were excluded $(\mathrm{n}=3)$. Urinary CXCL10 appeared to be elevated in isolated peritubular capillaritis versus glomerulitis $(P=0.04)$. There were no differences in serum CXCL10 $(P=0.50)$ (Figure 1C-D).

\section{Urinary CXCL10 Correlates With Specific Histological Compartments in an Independent Validation Set}

The independent validation set confirmed elevated urinary CXCL10 within the tubulointerstitial (tubulitis $\geq 2$ vs normal histology: median urinary CXCL10/creatinine [IQR], $1.27 \mathrm{ng} /$ mmol [0.21-4.48 ng/mmol] vs $0.25 \mathrm{ng} / \mathrm{mmol}[0.14-0.47 \mathrm{ng} /$ mmol]; $P=0.02$ ) and microvascular compartments (pure MVI vs normal histology: median urinary CXCL10/creatinine [IQR], $2.25 \mathrm{ng} / \mathrm{mmol}[0.96-5.73 \mathrm{ng} / \mathrm{mmol}]$ vs $0.25 \mathrm{ng} / \mathrm{mmol}$ [0.14-0.47 ng/mmol]; $P<0.0001$ ) (Figure $2 \mathrm{~A})$. Notably, urinary CXCL10 was not associated with isolated vascular inflammation (isolated $\mathrm{v}$ lesions vs normal histology: median urinary CXCL10/creatinine [IQR], $0.53 \mathrm{ng} / \mathrm{mmol}[0.26-2.44 \mathrm{ng} / \mathrm{mmol}]$ vs $0.25 \mathrm{ng} / \mathrm{mmol}[0.14-0.47 \mathrm{ng} / \mathrm{mmol}] ; P=0.06$ ) (Figure $2 \mathrm{~A}$ ).

The independent validation set also confirmed elevated urinary CXCL10 in patients with isolated peritubular capillaritis compared to isolated glomerulitis (pure peritubular capillaritis vs glomerulitis: median CXCL10/creatinine [IQR], $2.72 \mathrm{ng} /$ $\mathrm{mmol}[1.49-5.58 \mathrm{ng} / \mathrm{mmol}]$ vs $0.77 \mathrm{ng} / \mathrm{mmol}[0.16-1.69 \mathrm{ng} /$ mmol]; $P=0.02$ ) (Figure 2B). Patients with mixed MVI were excluded from this analysis $(n=13)$.

\section{Viral Infection as Confounders of Elevated Urinary and Serum CXCL10 Levels}

We sought to determine the extent to which active BKV and CMV infection affect urinary and serum CXCL10. In patients with normal histology and concomitant infection, urinary CXCL10 levels demonstrated a clear separation based on the type of viral infection (ie, BKV/normal histology vs CMV viremia/normal histology: median urinary CXCL10/ creatinine [IQR], $3.50 \mathrm{ng} / \mathrm{mmol}[1.97-13.0 \mathrm{ng} / \mathrm{mmol}]$ vs $0.67 \mathrm{ng} / \mathrm{mmol}[0.47-1.57 \mathrm{ng} / \mathrm{mmol}] ; P=0.02$; Figure $1 \mathrm{E})$. Urinary CXCL10 was similarly high in patients with BKV viremia and tubulitis $\geq 2$ (BKV viremia/normal histology vs tubulitis $\geq 2$ : median urinary CXCL10/creatinine [IQR], $3.50 \mathrm{ng} / \mathrm{mmol}[1.97-13.0 \mathrm{ng} / \mathrm{mmol}]$ vs $1.23 \mathrm{ng} / \mathrm{mmol}[0.48-$ $5.22 \mathrm{ng} / \mathrm{mmol}] ; P=0.14)$, whereas patients with $\mathrm{CMV}$ viremia/normal histology had similarly low urinary CXCL10 as normal histology/no infection patients (median urinary CXCL10/creatinine [IQR], $0.67 \mathrm{ng} / \mathrm{mmol}[0.47-1.57 \mathrm{ng} /$ $\mathrm{mmol}]$ vs $0.46 \mathrm{ng} / \mathrm{mmol}[0.32-1.31 \mathrm{ng} / \mathrm{mmol}] ; P=0.17)$.

Patients with normal histology and concomitant viral infection had the highest serum CXCL10 levels compared with the other groups $(P<0.0001$; Table 1 and Figure $1 \mathrm{~B})$. Notably, serum CXCL10 values were comparable among patients with BKV or CMV viremia $(P=0.44$; Figure $1 \mathrm{~F})$.

Finally, the independent validation set confirmed that urinary CXCL10 is elevated in BKV viremia, but not CMV viremia, in patients with normal histology (BKV vs CMV viremia: median urinary CXCL10/creatinine [IQR], $7.14 \mathrm{ng} / \mathrm{mmol}$ [2.10-18.6 ng/mmol] vs $1.47 \mathrm{ng} / \mathrm{mmol}[0.33-2.85 \mathrm{ng} / \mathrm{mmol}]$; $P=0.03$; Figure $2 \mathrm{C}$ ).

\section{DISCUSSION}

In this study, we characterized the impact of subclinical alloimmune and virus-related inflammation in different renal compartments with urinary and serum CXCL10. The 


\section{Urine}

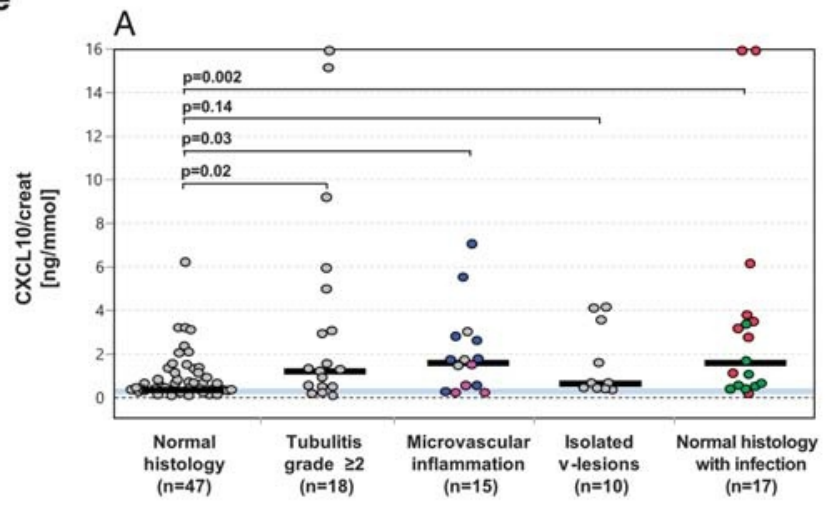

\section{Serum}

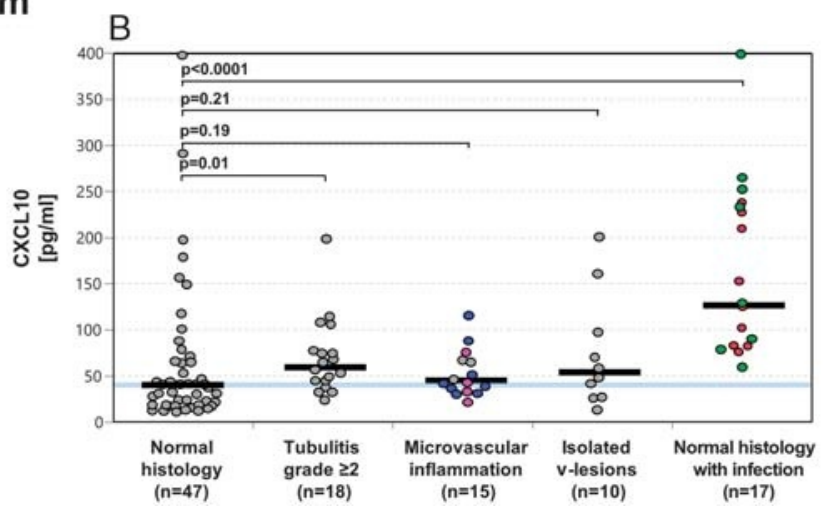

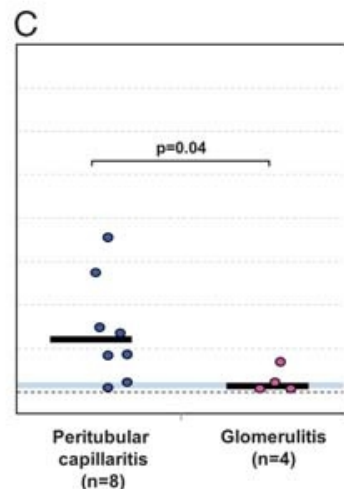

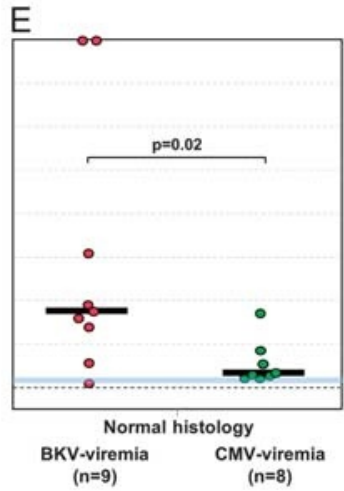

D

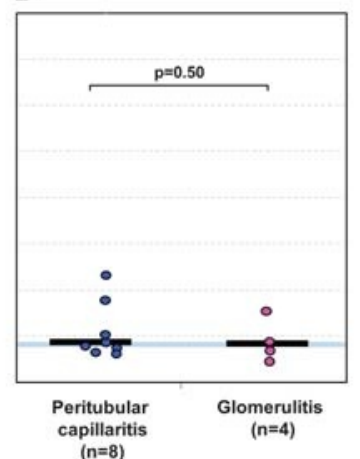

$\mathrm{F}$

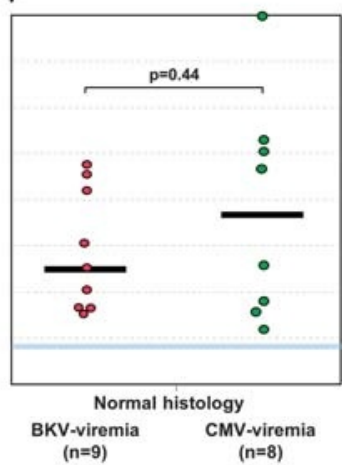

FIGURE 1. Correlation of urinary and serum CXCL10 levels with clinical-pathologic classification $(n=107)$. Urinary (A) and serum (B) CXCL10 levels among the 5 groups defined by compartment-specific inflammation +/- the presence of BKV/CMV-viremia are illustrated. Biopsies with pure MVI are further split into biopsies with only peritubular capillaritis (blue circles) or only glomerulitis (pink circles) and corresponding urinary/ serum CXCL10 levels illustrated (C and D, respectively). Biopsies with normal histology and concomitant infection are further split into biopsies with normal histology and BKV-viremia (red circles) or CMV-viremia (green circles) and corresponding urinary/serum CXCL10 levels illustrated (E and F, respectively). The vertical black bars indicate the median values of each group, the blue line indicates the median of the normal histology group, respectively.

principal findings of this study are that elevated urinary CXCL10 reflects subclinical alloimmune-mediated inflammation within the tubulointerstitial, but not the vascular/systemic compartments. The MVI group is notable in that elevated urinary CXCL10 was predominantly observed with isolated peritubular capillaritis, but not glomerulitis, further supporting the concept of compartment-restricted chemokine responses. These observations were also consistent with virus-specific inflammatory responses, where elevated urinary CXCL10 was observed in BKV (tubulointerstitial), but not CMV viremia (systemic). Finally, serum CXCL10 was not found to be a useful marker for subclinical (micro)vascular inflammation. Figure 3 summarizes conditions with or without elevated urinary CXCL10.

Currently, there are no noninvasive markers of MVI in routine use. Although de novo DSA may be an early marker for subclinical MVI, contention exists within the 2013 Transplantation Society HLA consensus guidelines regarding its use for routine posttransplant screening due to the high financial burden of implementing such a strategy. ${ }^{18}$ We have previously shown that pediatric renal transplant patients with isolated MVI have up to a 2.4-fold elevation of urinary CXCL10, suggesting this may be a potential noninvasive marker. ${ }^{14}$ These findings were confirmed in a prospective adult renal transplant cohort with clinically indicated biopsies, whereby urinary CXCL10 discriminated isolated (micro) vascular inflammation with an AUC of $0.70 .{ }^{15} \mathrm{~A}$ key finding of the present study was to extend these observations to an independent prospective, observational cohort of adult renal transplant patients with surveillance biopsies; thereby demonstrating that urinary CXCL10 outperforms serum creatinine and proteinuria by detecting subclinical MVI.

The urinary CXCL10 observations are also consistent with molecular diagnostic approaches that demonstrate a distinct interferon- $\gamma$ (IFNG) signature in renal allograft rejection, ${ }^{19,20}$ with CXCL10 identified as a top AMR classifier. ${ }^{21}$ Notably, IFNG-induced transcripts, such as CXCL9, CXCL10, and CXCL11, are highly expressed when T cell-mediated rejection (TCMR), AMR, and mixed rejection are compared with biopsies with other pathologies. ${ }^{22}$ This further supports our observation that elevated urinary CXCL10 levels may correlate with both subclinical TCMR and AMR.

An intriguing and novel observation was that urinary CXCL10 was elevated with isolated peritubular capillaritis, but not isolated glomerulitis. This subgroup analysis is limited by small numbers; but nevertheless, these results were reproducible within an independent validation population. Furthermore, these compartment-specific observations are highly consistent with those of Panzer et al, ${ }^{23}$ who demonstrated in a rat model of microvascular injury that CXCL10 was highly expressed in peritubular capillaries but not within glomeruli. Interestingly, Rabant et $\mathrm{al}^{15}$ reported urinary 
A

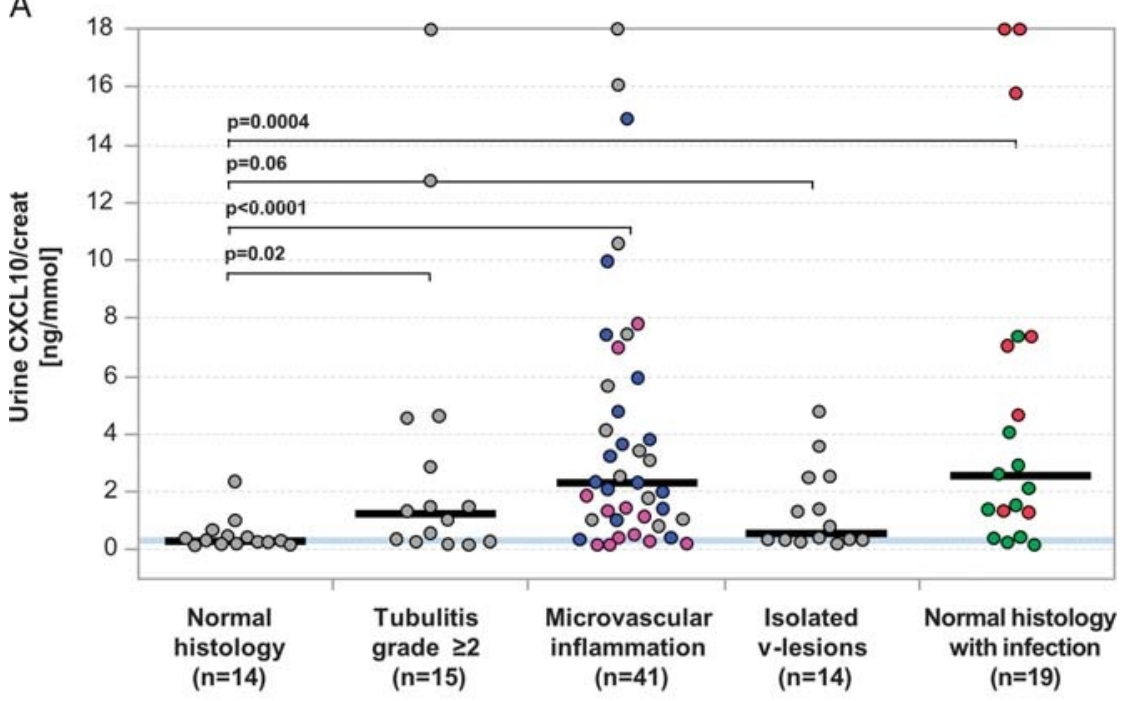

B
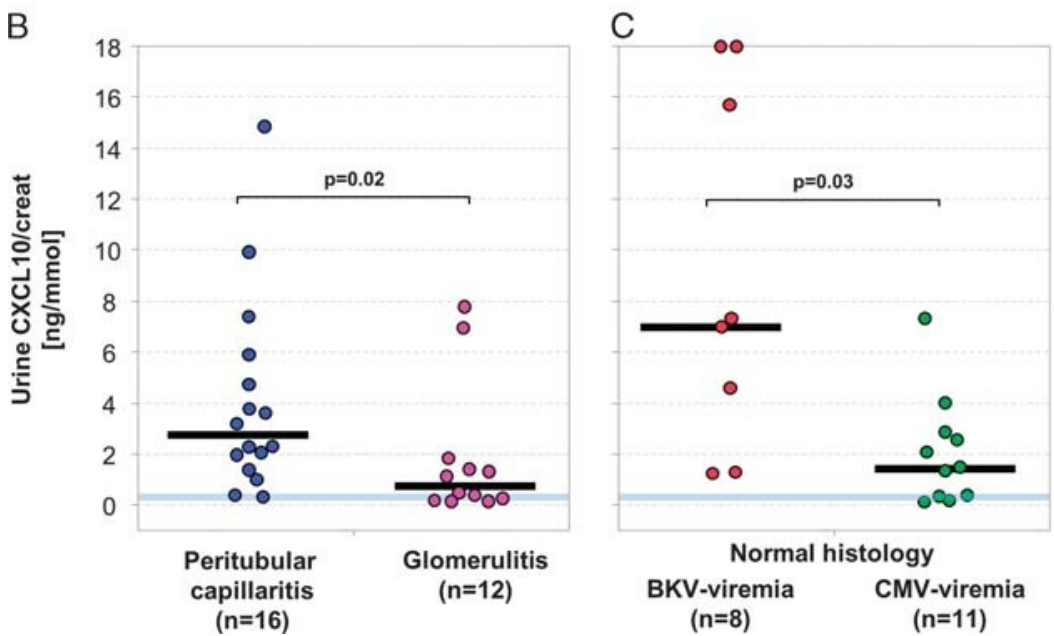

FIGURE 2. Correlation of urinary CXCL10 levels with clinical-pathologic classification $(n=103)$ in an independent validation set. Urinary CXCL10 levels among the 5 groups defined by compartment-specific inflammation +/- the presence of BKV/CMV-viremia are illustrated (A). Biopsies with pure MVI are further split into biopsies with only peritubular capillaritis (blue circles) or only glomerulitis (pink circles) and corresponding urinary CXCL10 levels are illustrated (B). Biopsies with normal histology and concomitant infection are further split into biopsies with normal histology and BKV-viremia (red circles) or CMV-viremia (green circles) and corresponding urinary CXCL10 levels illustrated (C). The vertical black bars indicate the median values of each group, the blue line indicates the median of the normal histology group, respectively.

CXCL10 as a marker of pure clinical AMR (AUC, 0.70) in the absence of tubulointerstitial inflammation. Taken together, these data suggest that the diagnostic performance of urinary CXCL10 is better for subclinical/clinical peritubular capillaritis predominant phenotypes versus glomerulitis.

Patients with early isolated $\mathrm{v}$ lesions (within the first year posttransplant) did not demonstrate elevated urinary CXCL10, and there are several possibilities for this. First, the anatomical relationship of the isolated $\mathrm{v}$ lesions may not be sufficiently proximate for CXCL10 to be detectable in the urine. Alternatively, CXCL10 may not be upregulated in patients with isolated v lesions. Controversy exists regarding the prognostic significance of isolated $\mathrm{v}$ lesions ${ }^{24,25}$ because some early isolated $\mathrm{v}$ lesions do not meet the molecular classification score for TCMR or AMR, and these do not impact graft survival. ${ }^{24}$ Indeed, early isolated v lesions may reflect peritransplant endothelial injury, with a better prognosis than alloimmune-related $\mathrm{v}$ lesions. ${ }^{24}$ Isolated $\mathrm{v}$ lesions also have a highly heterogeneous inflammatory cell infiltrate suggesting that different pathogenic processes may be present. $^{26}$ Elevated urinary CXCL10 is an early prognostic marker for graft loss, late acute rejection, and graft dysfunction, ${ }^{27}$ and the low urinary CXCL10 levels in our patients with early isolated $\mathrm{v}$ lesions suggest they may have a better graft prognosis. Taken together, these data suggest that isolated $\mathrm{v}$ lesions may have multiple pathophysiological pathways with different long-term outcomes, and it is possible that urinary CXCL10 may help to identify isolated $\mathrm{v}$ lesions with a better overall prognosis. Previous studies which report an association between urinary CXCL10 and MVI did not evaluate isolated v lesions, ${ }^{14,15}$ and only 2 of the 24 patients developed isolated $\mathrm{v}$ lesions 12 months posttransplant in this study. Therefore, these findings remain to be clarified in patients with late alloimmune-mediated isolated $\mathrm{v}$ lesions.

Interestingly, CMV infection, which can affect circulating hematopoietic cells and potentially vascular endothelial cells, led to elevated serum CXCL10, but not urine. These findings are consistent with the lack of urinary CXCL9 elevation in 


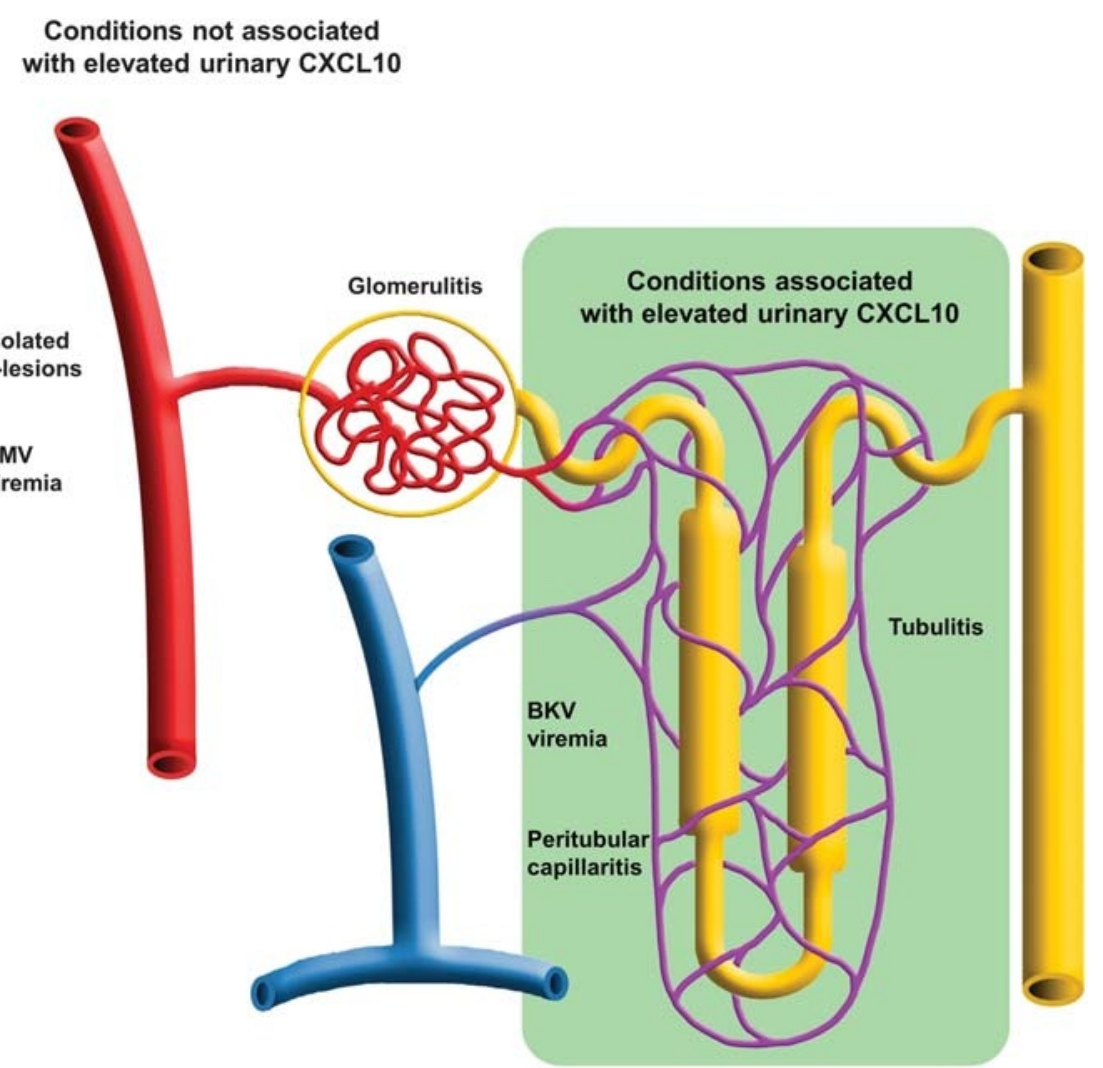

FIGURE 3. Schematic of conditions associated with or without elevated urinary CXCL10.

CMV infection observed by Hauser et al. ${ }^{9}$ In contrast BKV infection, which targets tubular epithelial cells with subsequent hematogenic spreading, induced both elevated serum and urinary CXCL10 levels. This is consistent with virusspecific inflammatory responses in different compartments. Furthermore, the novel observation of elevated urinary CXCL10 levels in the setting of BKV viremia with concomitant normal histology is intriguing. This raises the possibility that normal histology might result from sampling error and patients may have early subclinical BKV nephropathy with tubulointerstitial inflammation that is detectable by urinary CXCL10 and BKV PCR alone. ${ }^{28}$

As elevated pretransplant serum CXCL10 has been shown to be an early predictor for acute rejection and graft loss, ${ }^{29,30}$ we sought to delineate the associations of posttransplant serum CXCL10 with underlying subclinical histology. We hypothesized that serum CXCL10 would correspond with inflammation in the microvascular and vascular compartments. However, serum CXCL10 was not a robust marker of subclinical (micro)vascular inflammation.

The tubulitis $\geq 2$ group had increased overall inflammation compared to the pure MVI and isolated $\mathrm{v}$ lesion groups, as there was concomitant interstitial inflammation in the tubulitis $\geq 2$ group. These highly restricted subclinical phenotypes were able to elucidate associations of urinary CXCL10 with specific renal compartments; however the small numbers preclude multivariate analysis to determine if they are independent. Nevertheless, these findings are consistent with Rabant et al, who demonstrated that Banff $i$, $t$, and ptc scores were significantly and independently associated with urinary CXCL10, whereas glomerulitis was not. ${ }^{15}$
In conclusion, urinary CXCL10 is associated with alloimmune and virus compartment-specific inflammation. We independently confirmed that urinary CXCL10 is associated with MVI, and extended these findings to subclinical MVI. Clearly, this is a highly selected cohort, suggesting that its diagnostic performance may be modest; nevertheless, these findings are consistent with Rabant et al. ${ }^{15}$ The elevation of urinary CXCL10 in MVI was predominantly related to peritubular capillaritis, and this may be due to the close proximity of the peritubular capillaries to the urine.

\section{ACKNOWLEDGMENTS}

The authors thank the staff of the University Hospital Basel Renal Transplant Unit and the histocompatibility laboratory for collection and processing of serum and urine samples.

\section{REFERENCES}

1. Moreso F, Ibernon M, Goma M, et al. Subclinical rejection associated with chronic allograft nephropathy in protocol biopsies as a risk factor for late graft loss. Am J Transplant. 2006;6:747-752.

2. Heilman RL, Devarapalli Y, Chakkera HA, et al. Impact of subclinical inflammation on the development of interstitial fibrosis and tubular atrophy in kidney transplant recipients. Am J Transplant. 2010;10:563-570.

3. Park WD, Griffin MD, Cornell LD, et al. Fibrosis with inflammation at one year predicts transplant functional decline. J Am Soc Nephrol. 2010;21: 1987-1997.

4. Wiebe C, Gibson IW, Blydt-Hansen TD, et al. Evolution and clinical pathologic correlations of de novo donor-specific HLA antibody post kidney transplant. Am J Transplant. 2012;12:1157-1167.

5. Loupy A, Suberbielle-Boissel C, Hill GS, et al. Outcome of subclinical antibody-mediated rejection in kidney transplant recipients with preformed donor-specific antibodies. Am J Transplant. 2009;9:2561-2570. 
6. Wilczek HE. Percutaneous needle biopsy of the renal allograft. A clinica safety evaluation of 1129 biopsies. Transplantation. 1990;50:790-797.

7. Hricik DE, Nickerson P, Formica RN, et al. Multicenter validation of urinary CXCL9 as a risk-stratifying biomarker for kidney transplant injury. Am J Transplant. 2013;13:2634-2644.

8. Matz M, Beyer J, Wunsch D, et al. Early post-transplant urinary IP-10 expression after kidney transplantation is predictive of short- and long-term graft function. Kidney Int. 2006;69:1683-1690.

9. Hauser IA, Spiegler S, Kiss E, et al. Prediction of acute renal allograft rejection by urinary monokine induced by IFN-gamma (MIG). J Am Soc Nephrol. 2005;16:1849-1858.

10. Schaub S, Nickerson P, Rush D, et al. Urinary CXCL9 and CXCL10 levels correlate with the extent of subclinical tubulitis. Am J Transplant. 2009;9: 1347-1353.

11. Jackson JA, Kim EJ, Begley B, et al. Urinary chemokines CXCL9 and CXCL10 are noninvasive markers of renal allograft rejection and BK viral infection. Am J Transplant. 2011;11:2228-2234.

12. Ho J, Rush DN, Karpinski M, et al. Validation of urinary CXCL10 as a marker of borderline, subclinical, and clinical tubulitis. Transplantation. 2011;92:878-882.

13. Hirt-Minkowski P, Amico P, Ho J, et al. Detection of clinical and subclinical tubulo-interstitial inflammation by the urinary CXCL10 chemokine in a reallife setting. Am J Transplant. 2012;12:1811-1823.

14. Blydt-Hansen TD, Gibson IW, Gao A, et al. Elevated urinary CXCL10-tocreatinine ratio is associated with subclinical and clinical rejection in pediatric renal transplantation. Transplantation. 2015;99:797-804.

15. Rabant M, Amrouche L, Lebreton X, et al. Urinary C-X-C motif dhemokine 10 independently improves the noninvasive diagnosis of antibody-mediated kidney allograft rejection. J Am Soc Nephrol. 2015;26:2840-2851.

16. Amico $P$, Hirt-Minkowski $P$, Honger $G$, et al. Risk stratification by the virtual crossmatch: a prospective study in 233 renal transplantations. Transp/ Int. 2011;24:560-569.

17. Haas M, Sis B, Racusen LC, et al. Banff 2013 meeting report: inclusion of c4d-negative antibody-mediated rejection and antibody-associated arterial lesions. Am J Transplant. 2014;14:272-283.

18. Tait BD, Süsal C, Gebel HM, et al. Consensus guidelines on the testing and clinical management issues associated with HLA and non-HLA antibodies in transplantation. Transplantation. 2013;95:19-47.
19. Sellares J, Reeve J, Loupy A, et al. Molecular diagnosis of antibodymediated rejection in human kidney transplants. Am J Transplant. 2013; 13:971-983

20. Sigdel TK, Bestard O, Tran TQ, et al. A computational gene expression score for predicting immune injury in renal allografts. PLoS One. 2015; 10:e0138133.

21. Venner JM, Hidalgo LG, Famulski KS, et al. The molecular landscape of antibody-mediated kidney transplant rejection: evidence for NK involvement through CD16a Fc receptors. Am J Transplant. 2015;15: $1336-1348$

22. Halloran PF, Famulski K, Reeve J. The molecular phenotypes of rejection in kidney transplant biopsies. Curr Opin Organ Transplant. 2015; 20:359-367.

23. Panzer U, Steinmetz OM, Reinking RR, et al. Compartment-specific expression and function of the chemokine IP-10/CXCL10 in a model of renal endothelial microvascular injury. J Am Soc Nephrol. 2006;17:454-464.

24. Salazar ID, Merino LM, Chang J, et al. Reassessing the significance of intimal arteritis in kidney transplant biopsy specimens. J Am Soc Nephrol. 2015;26:3190-3198.

25. Wu KY, Budde K, Schmidt D, et al. Acute cellular rejection with isolated v lesions is not associated with more favorable outcomes than vascular rejection with more tubulointerstitial inflammations. Clin Transplant. 2014; 28:410-418

26. Lim BJ, Kwon HJ, Bae YS, et al. Immunohistochemical analysis of infiltrating inflammatory cells in the isolated v-lesion of allograft kidney. Transplant Proc. 2015;47:622-625.

27. Hirt-Minkowski P, Ho J, Gao A, et al. Prediction of long-term renal allograft outcome by early urinary CXCL10 chemokine levels. Transplant Direct. 2015;1:e31.

28. Menter T, Mayr M, Schaub S, et al. Pathology of resolving polyomavirusassociated nephropathy. Am J Transplant. 2013;13:1474-1483.

29. Rotondi M, Rosati A, Buonamano A, et al. High pretransplant serum levels of CXCL10/IP-10 are related to increased risk of renal allograft failure. Am J Transplant. 2004:4:1466-1474.

30. Lazzeri E, Rotondi M, Mazzinghi B, et al. High CXCL10 expression in rejected kidneys and predictive role of pretransplant serum CXCL10 for acute rejection and chronic allograft nephropathy. Transplantation. 2005; 79:1215-1220. 